\title{
MANTA versus ProGlide vascular closure devices in transfemoral transcatheter aortic valve implantation
}

Fausto Biancari, ${ }^{\mathrm{a}, \mathrm{b}}$ Hannu Romppanen, ${ }^{\mathrm{c}}$ Mikko Savontaus, ${ }^{\mathrm{a}}$ Antti Siljander, ${ }^{\mathrm{c}}$ Timo Mäkikallio, ${ }^{\mathrm{d}}$ Olli-Pekka Piira, ${ }^{\mathrm{d}}$ Jarkko Piuhola, ${ }^{\mathrm{d}}$ Viivi Vilkki, ${ }^{\mathrm{a}}$ Antti Ylitalo, ${ }^{\mathrm{a}}$ Tuija Vasankari, ${ }^{a}$ Juhani K.E. Airaksinen, ${ }^{a}$ Matti Niemelä ${ }^{\mathrm{d}}$

${ }^{a}$ Heart Center, Turku University Hospital and University of Turku, Turku, Finland;

${ }^{\mathrm{b}}$ Department of Surgery, University of Oulu, Oulu, Finland;

${ }^{\mathrm{c}}$ Heart Center, Heart Center, Kuopio University Hospital, Kuopio, Finland

${ }^{\mathrm{d}}$ Department of Cardiology, Oulu University Hospital, Oulu, Finland.

Footnote: All the above listed authors take responsibility for all aspects of the reliability and freedom from bias of the data presented and their discussed interpretation.

For correspondence:

Prof. Fausto Biancari, Heart Center,

Turku University Hospital, Hämeentie 11, PL 52, 20521 Turku, Finland

Tel.: +358 407333973

E-mail: fausto.biancari@yahoo.it

Grant support: None

Conflicts of interest: None

Keywords: Vascular closure device; MANTA, ProGlide; TAVI.

Text word count: 1491 words

Abstract word count: 200 words 


\title{
HIGHLIGHTS
}

- The safety and efficacy of currently available vascular closure devices (VCD) is debated.

- This study showed that the risk of vascular injury during transfemoral transcatheter aortic valve implantation is substantial even when using VCDs for large-bore access.

- The MANTA VCD may be not inferior to the ProGlide VCD and may significantly reduce the need of additional VCDs for completion of hemostasis.

\begin{abstract}
Background: The MANTA system is a novel vascular closure device (VCD) and its safety and efficacy were compared to the ProGlide VCD in patients undergoing transfemoral transcatheter aortic valve implantation (TAVI).
\end{abstract}

Methods: This is a retrospective study including 222 patients who underwent transfemoral TAVI at three Finnish University Hospitals. The MANTA VCD was used in 107 patients and their outcome was compared with that of 115 patients in whom the arterial access was closed with the ProGlide VCD.

Results: VARC-2 VCD failure occurred less frequently in the MANTA cohort (3.7\% vs. 7.8\%, $\mathrm{p}=0.378$ ), but the difference did not reach statistical significance. When adjusted for the introducer outer diameter, the MANTA cohort had similar rates of VARC-2 major vascular complications ( $9.3 \%$ vs. $12.2 \%$, adjusted: $\mathrm{p}=0.456$ ), VARC-2 life-threatening/disabling bleeding ( $9.3 \%$ vs. $6.1 \%$, adjusted: $\mathrm{p}=0.296$ ) and need of invasive treatment of bleeding ( $4.7 \%$ vs. $7.0 \%$, adjusted: $\mathrm{p}=0.416$ ) compared to the ProGlide cohort. Additional VCDs were more frequently needed in the ProGlide cohort (58.3\% vs. $1.9 \%, \mathrm{p}<0.0001)$. 
Conclusions: In patients undergoing transfemoral TAVI, the MANTA VCD showed a similar risk of VARC-2 vascular and bleeding complications compared to the ProGlide VCD, but it reduced significantly the need of additional VCDs for completion of hemostasis.

\section{Introduction}

Advances of transcatheter technology is limited by the need of large-bore access, which is associated with a significant risk of vascular access complications [1]. Vascular closure devices (VCDs) are used to reduce such complications after transfemoral transcatheter aortic valve

implantation (TAVI) [2]. Still the rate of vascular injury at access site remains substantial [1]. In this study we evaluated the efficacy and safety of a novel VCD, the MANTA system (Essential Medical Inc., Malvern, Pennsylvania, USA) in patients undergoing TAVI.

\section{Methods}

The data of 222 consecutive patients who underwent transfemoral TAVI using a MANTA or ProGlide VCDs at three Finnish University Hospitals (Kuopio, Oulu and Turku, Finland) from September 2016 to October 2017 was retrospectively reviewed. The MANTA VCD was used in 107 patients after TAVI and their outcome was compared with that of 115 patients in whom the arterial access was closed with the ProGlide VCD (Abbott Vascular, Abbott Park, Illinois, USA), 
which is considered one of the most effective large-bore VCDs [3]. This study was approved by the Institutional Board of each participating center.

TAVI procedures and access sites were planned after evaluation of computed tomography scans. The femoral arteries were approached under fluoroscopy or ultrasound guidance.

The ProGlide VCD is a 6-F, suture-mediated closure system designed for closing the arterial access site in patients undergoing catheterisation procedures. Two ProGlide VCDs were deployed at the beginning of the procedure into the common femoral artery with an angle of 45$60^{\circ}$ at $2 \mathrm{o}^{\prime}$ clock and $10 \mathrm{o}^{\prime}$ clock position, respectively, as described elsewhere [4]. If a third ProGlide VCD was needed, this was deployed to 12 o'clock position. The MANTA system is a 14-F and 18-F VCD for closing arteriotomies of 10 to $14 \mathrm{~F}$ and 15 to 22 F sheaths, respectively. This VCD consists of a resorbable polymer intra-arterial toggle connected to an extra-arterial hemostatic bovine collagen pad by a non-resorbable polyester suture and secured with a stainless-steel suture lock. This VCD was deployed according to previously described instruction [5]. Shortly, after the common femoral artery was accessed, an 8-F puncture dilator was inserted to evaluate the distance from skin to the inner side of the femoral artery wall. When the TAVI procedure was accomplished, the procedural sheath was exchanged for the MANTA sheath and the closure unit inserted and withdrawn up to the predetermined deployment level. The toggle was released, the assembly component withdrawn, and the collagen pad secured onto the outer arterial wall by the stainless-steel lock. After hemostasis was achieved, the suture was cut at skin level and the skin incision was closed with a resorbable intracutaneous suture. 
VCDs were deployed by seven senior cardiologists with large experience in TAVI procedures. Each cardiologist deployed their first five to ten MANTA devices under the guidance of an Essential Medical's technical advisor.

The main outcome measures of this study were the need of invasive treatment of bleeding, lifethreatening/disabling bleeding and major vascular complications according to the VARC-2 definition criteria [6]. The secondary outcomes were hospital/ 30-day mortality, stroke, VARC-2 VCD failure, VARC-2 major bleeding, surgical treatment for bleeding, red blood cell transfusion, postoperative decrease of hemoglobin, additional use of VCDs, VARC-2 minor vascular complications, femoral/iliac arteries stenosis or dissection, lower limb ischemia, access site infection, irreversible nerve injury and any access site complication at 1-month follow-up. Furthermore, an analysis was performed to compare the direct costs of the VCDs used, and it did not consider the postoperative outcome related to these devices.

Statistical analyses were performed using the SPSS v. 24.0 statistical software (IBM

Corporation, NY, USA). Mann-Whitney, Fisher's and Chi-square tests were used for univariate analysis. The effect of VCD on the outcomes was adjusted for the introducer outer diameter in logistic regression. All tests were two-sided and $\mathrm{p}<0.05$ was set for statistical significance.

\section{Results}

Patients' characteristics and procedural data are summarized in Table 1. The study cohorts were balanced in terms of baseline covariates as confirmed by similar EuroSCORE II (ProGlide, $4.4 \pm 3.2 \%$ vs. MANTA, $4.4 \pm 3.7 \%, \mathrm{p}=0.806)$. The implanted TAVI devices significantly differed between the study cohorts $(\mathrm{p}<0.0001)$ and the introducer outer diameter was larger in the ProGlide cohort $(\mathrm{p}<0.0001)$. 
At completion of vascular access closure, vascular status of the access site was verified in 215 patients $(96.8 \%$, by ultrasound in $29.7 \%$ and by angiography in $67.1 \%$ of cases $)$.

The early outcomes in the study cohorts are summarized in Table 2. VCD failure occurred less frequently in the MANTA cohort (3.7\% vs. $7.8 \%$, adjusted: $\mathrm{p}=0.238$ ), but the difference did not reach statistical significance (Tab. 1). VARC-2 major vascular complications (9.3\% vs. 12.2\%, adjusted for the introducer outer diameter: $\mathrm{p}=0.456)$, VARC-2 life-threatening/disabling bleeding (9.3\% vs. $6.1 \%$, adjusted for the introducer outer diameter: $\mathrm{p}=0.296)$ and need of invasive treatment of bleeding ( $4.7 \%$ vs. $7.0 \%$, adjusted for the introducer outer diameter: $\mathrm{p}=0.416)$ from the index access site were similar in the MANTA and ProGlide device cohorts (Tab. 2). Additional VCDs were more frequently needed in the ProGlide cohort (58.3\% vs. 1.9\%, $\mathrm{p}<0.0001)$.

The cost of each device at these participating centers was $950 €(1172 \$)$ for the MANTA system, $169 €(209 \$)$ for the ProGlide system and $124 €(153 \$)$ for the Angioseal system. When any additional VCD was considered in this analysis, the mean total costs of VCDs was $968 \pm 129$ $€(1194 \pm 159 \$)$ in the MANTA cohort and 433 $\pm 105 €(533 \pm 129 \$)$ in the ProGlide cohort $(\mathrm{p}<0.0001)$.

\section{Discussion}

This study showed that the risk of vascular injury is substantial even when using third generation TAVI prostheses with currently available VCDs for large-bore access. The present results showed that the novel MANTA closure device is associated with a somewhat lower rate of VCD failure and a significantly lower rate of additional VCDs as compared to ProGlide VCD, which is considered one of the safest of such devices [3]. The risk of VARC-2 bleeding and major 
vascular complications was similar between the study cohorts even when adjusted for the outer diameter of the introducer. However, the severity of bleeding in this patient population is not uniquely related to the TAVI access site, whereas the rate of any vascular complication and invasive treatment was numerically lower in the MANTA cohort. Despite the limited size and non-randomized nature of this study, the present results suggest that the MANTA device may be not inferior to the ProGlide device and may significantly reduce the need of additional VCDs for completion of hemostasis. Although the use of additional VCDs may be related to operator's policy, in this series the same operators deployed either the MANTA or ProGlide VCDs. This likely decreased the risk of bias of using additional VCD. Still, the need of additional VCDs was much larger in the ProGlide cohort (58.3\% vs. $1.9 \%, \mathrm{p}<0.0001)$.

Analysis of costs related to the price of these VCDs showed that the MANTA device was significantly more expensive than the ProGlide device. Although the MANTA device is rather easy-to-use, the lack of data on device implanting times, the timing of mobilization and discharge to home prevents reliable cost-benefit analysis. Furthermore, the increased costs and the learning curve of any novel medical device prevent at this stage conclusive results of the cost-benefit ratio of using the MANTA compared to the ProGlide VCD in patients undergoing TAVI.

\section{Conclusions}

These preliminary results suggest that the MANTA system is a rather easy-to-use VCD, which promptly achieves hemostasis at the femoral artery access after TAVI. However, the use of large-bore catheters is still associated with significant vascular complications despite the use of this novel VCD. In view of the limited size of this comparative analysis and the possible impact 
of an initial learning curve with the use of the MANTA system, further studies of larger size are needed to prove the efficacy and safety of this device.

\section{References}

1. H. Kawashima, Y. Watanabe, K. Kozuma, et al. Propensity-matched comparison of percutaneous and surgical cut-down approaches in transfemoral transcatheter aortic valve implantation using a balloon-expandable valve. EuroIntervention 12 (16) (2017) pp. 19541961.

2. N.M. Van Mieghem, A. Latib, J. van der Heyden, et al. Percutaneous plug-based arteriotomy closure device for large-bore access: a multicenter prospective study. JACC Cardiovasc. Interv. 10 (6) (2017) pp. 613-619.

3. Z. Dimitriadis, W. Scholtz, J. Börgermann, et al. Impact of closure devices on vascular complication and mortality rates in TAVI procedures. Int. J. Cardiol. 241 (2017) pp. 133137.

4. S. Toggweiler, J. Leipsic, R.K. Binder, et al. Management of vascular access in transcatheter aortic valve replacement: part 1: basic anatomy, imaging, sheaths, wires, and access routes. JACC. Cardiovasc. Interv. 6 (7) (2013) pp. 643-653.

5. L. van Gils, J. Daemen, G. Walters, et al. MANTA, a novel plug-based vascular closure device for large bore arteriotomies: technical report. EuroIntervention 12 (7) (2016) pp. 896900.

6. A.P. Kappetein, S.J. Head, P. Généreux, et al. Updated standardized endpoint definitions for transcatheter aortic valve implantation: the Valve Academic Research Consortium-2 consensus document (VARC-2). Eur. J. Cardiothorac. Surg. 42 (5) (2012) pp. S45-60. 
Table 1. Baseline characteristics and procedural data.

\begin{tabular}{|c|c|c|c|}
\hline & $\begin{array}{l}\text { ProGlide device } \\
115 \mathrm{pts}\end{array}$ & $\begin{array}{l}\text { MANTA device } \\
107 \text { pts }\end{array}$ & p-value \\
\hline Age (years) & $80.7 \pm 6.8$ & $79.8 \pm 6.0$ & 0.189 \\
\hline Female & $63(54.8)$ & $66(61.7)$ & 0.298 \\
\hline Body mass index $\left(\mathrm{kg} / \mathrm{m}^{2}\right)$ & $28.0 \pm 5.0$ & $27.3 \pm 4.8$ & 0.376 \\
\hline Hemoglobin $(g / L)$ & $127 \pm 14$ & $126 \pm 16$ & 0.351 \\
\hline Platelet count $\left(10^{9} / \mathrm{L}\right)$ & $211 \pm 80$ & $222 \pm 57$ & 0.043 \\
\hline Creatinine $(\mathrm{mmol} / \mathrm{l})$ & $96 \pm 52$ & $109 \pm 74$ & 0.017 \\
\hline Dialysis & $1(0.9)$ & $3(2.8)$ & 0.354 \\
\hline Atrial fibrillation & $40(34.8)$ & $42(39.3)$ & 0.491 \\
\hline Diabetes & $30(26.1)$ & $27(25.2)$ & 0.884 \\
\hline Coronary artery disease & $61(53.0)$ & $60(56.1)$ & 0.650 \\
\hline Stroke & $14(12.2)$ & $7(6.5)$ & 0.152 \\
\hline Pulmonary disease & $24(20.9)$ & $22(20.6)$ & 0.955 \\
\hline Extracardiac arteriopathy & $11(9.6)$ & $10(9.3)$ & 0.955 \\
\hline $\begin{array}{l}\text { Prior percutaneous coronary } \\
\text { intervention }\end{array}$ & $25(21.7)$ & $29(27.1)$ & 0.352 \\
\hline Prior cardiac surgery & $17(14.8)$ & $11(10.3)$ & 0.313 \\
\hline $\begin{array}{l}\text { Prior vascular procedure on the } \\
\text { femoral arteries }\end{array}$ & $3(2.6)$ & $1(0.9)$ & 0.349 \\
\hline Access site calcification & $78(67.8)$ & $66(61.7)$ & 0.338 \\
\hline High femoral a. bifurcation & $11(9.6)$ & $11(10.3)$ & 0.859 \\
\hline \multicolumn{4}{|l|}{ Preop. antithrombotics } \\
\hline Aspirin & $55(47.8)$ & $62(57.9)$ & 0.131 \\
\hline New oral anticoagulants & $7(6.1)$ & $9(8 . .4)$ & 0.503 \\
\hline Warfarin & $38(33.0)$ & $32(29.9)$ & 0.615 \\
\hline 2PY12 inhibitor & $14(12.2)$ & $13(12.3)$ & 0.984 \\
\hline EuroSCORE II (\%) & $4.4 \pm 3.2$ & $4.4 \pm 3.7$ & 0.806 \\
\hline TAVI device & & & $<0.0001$ \\
\hline Acurate Neo & $19(16.5)$ & $26(24.3)$ & \\
\hline Size (mm) & $26.0 \pm 1.5$ & $25.5 \pm 1.5$ & 0.2141 \\
\hline Evolut R & $13(11.3)$ & $30(28.0)$ & \\
\hline Size $(\mathrm{mm})$ & $29.9 \pm 3.1$ & $30.5 \pm 3.1$ & 0.557 \\
\hline Portico & 0 & $3(2.8)$ & \\
\hline Size $(\mathrm{mm})$ & - & $26.0 \pm 1.0$ & - \\
\hline Sapien 3 & $83(72.2)$ & $48(44.9)$ & \\
\hline Size $(\mathrm{mm})$ & $25.4 \pm 2.3$ & $25.3 \pm 2.4$ & 0.718 \\
\hline Introducer size (mm) & $15.2 \pm 1.6$ & $15.7 \pm 1.9$ & 0.028 \\
\hline Introducer outer diameter (mm) & $23.0 \pm 1.4$ & $21.9 \pm 2.0$ & $<0.0001$ \\
\hline \multicolumn{4}{|l|}{ Manta device size } \\
\hline $14-\mathrm{F}$ & - & $9(8.4)$ & - \\
\hline $18-\mathrm{F}$ & - & 98 (91.6) & - \\
\hline
\end{tabular}

Continuous variables are reported as means and standard deviation and nominal variables as counts and percentages. TAVI: transcatheter aortic valve implantation; 
Table 2. Outcomes

\begin{tabular}{|c|c|c|c|}
\hline & $\begin{array}{c}\text { ProGlide device } \\
115 \mathrm{pts}\end{array}$ & $\begin{array}{c}\text { MANTA device } \\
107 \text { pts }\end{array}$ & p-value \\
\hline In-hospital death & $1(0.9)$ & 0 & 1.000 \\
\hline 30-day mortality & $2(1.7)$ & 0 & 0.498 \\
\hline VCD-related death & 0 & 0 & - \\
\hline Stroke & $1(0.9)$ & 0 & 1.000 \\
\hline VCD failure (VARC-2) & $8(7.8)$ & $4(3.7)$ & 0.378 \\
\hline VARC 2 bleeding & & & 0.659 \\
\hline Life-treatening/disabling & $7(6.1)$ & $10(9.3)$ & \\
\hline Major & $19(16.5)$ & $17(15.9)$ & \\
\hline Bleeding requiring invasive treatment & $8(7.0)$ & $5(4.7)$ & 0.469 \\
\hline Surgery for bleeding & $5(4.3)$ & $4(3.7)$ & 1.000 \\
\hline $\mathrm{RBC}$ transfusion & $10(8.8)$ & $14(13.1)$ & 0.388 \\
\hline $\mathrm{RBC}$ units transfused (if required) & $1.5 \pm 1.6$ & $2.4 \pm 1.7$ & 0.752 \\
\hline$\Delta$-hemoglobin & $19 \pm 13$ & $20 \pm 12$ & 0.728 \\
\hline Additional use of VCD & $67(58.3)$ & $2(1.9)$ & $<0.0001$ \\
\hline Angioseal & $52(45.2)$ & 0 & \\
\hline ProGlide & $19(16.5)$ & 0 & \\
\hline Manta & 0 & $2(1.9)$ & \\
\hline Major vascular complications (VARC-2) & $14(12.2)$ & $10(9.3)$ & 0.498 \\
\hline Minor vascular complications (VARC-2) & $3(2.6)$ & $4(3.7)$ & 0.630 \\
\hline Femoral/iliac artery stenosis/dissection & $6(5.2)$ & $5(4.7)$ & 0.652 \\
\hline VCD distal embolization & 0 & 0 & - \\
\hline Lower limb ischemia & 0 & $1(0.9)$ & 0.482 \\
\hline Access site infection & 0 & 0 & - \\
\hline Irreversible nerve injury & 0 & 0 & - \\
\hline \multicolumn{4}{|l|}{ Access-site complications at 1-month* } \\
\hline Bleeding & $1(0.9)$ & 0 & 1.000 \\
\hline
\end{tabular}

Continuous variables are reported as mean and standard deviation. Categorical variables are reported as absolute number and percentages (in parentheses). *: data not available in four patients. VCD: vascular closure device; VARC-2: Valve Academic Research Consortium-2; RBC: red blood cell. 